\title{
Pulsar timing measurements with the 32-m TCfA radiotelescope
}

\author{
W. Lewandowski, M. Konacki, M. Redmerska, G. Feiler \\ Toruń Centre for Astronomy, Nicolaus Copernicus University, \\ 87-100 Toruń, Gagarina 11, Poland
}

\author{
A. Wolszczan \\ Penn State University, Department of Astronomy and Astrophysics, 525 \\ Davey Laboratory, University Park, PA 16802, USA \\ Toruń Centre for Astronomy, Nicolaus Copernicus University \\ ul. Gagarina 11, 87-100 Torun, Poland
}

Accurate, long-term timing measurements of pulsars provide a powerful method to study a variety of astrophysical phenomena. For "normal", slow pulsars, the dominant factors that limit the timing precision are the intrinsic timing noise and single pulse "jitter" (e.g. Cordes 1993). In fact, because the pulse jitter surpasses radiometer noise for sufficiently strong pulsars and no further improvement of the timing precision can be achieved by increasing the antenna gain, the timing of such sources can be very efficiently conducted with suitably equipped medium-size radiotelescopes.

We have been timing slow pulsars with the 32-m TCfA radiotelescope in Toruń, Poland, since July 1996, using a dual-channel, circular polarization Lband receiving system at frequencies around $1.7 \mathrm{GHz}$, and a $2 \times 64 \times 3 \mathrm{MHz}$ channel pulsar backend, the Penn State Pulsar Machine - 2 (PSPM-2; for more details, see Konacki et al. 1999). Our gradually expanding source list currently includes 88 pulsars timed once a week with a millisecond precision using the observatory's UTC-calibrated H-maser clock. Data analysis is routinely performed with the TEMPO ${ }^{1}$ software package. With a particularly dense, weekly sampling and a $\leq 1 \mathrm{~ms}$ timing precision, the TCfA program has a sensitivity to detect previously overlooked short period, low orbital inclination binaries, as well as very low-mass, planetary companions. In addition, it will be very useful in identifying and monitoring pulsar timing glitches and other forms of the timing noise.

Two years of timing of 38 pulsars from our observing list have already produced interesting limits on possible terrestrial-mass companions to some of these objects. The planetary limits, expressed in terms of the orbital period and the planet mass for circular, edge-on orbits, are shown in Fig. 1a. Limiting masses were calculated using the rms residuals left over from the best fits of timing models to data. The models included the pulsar period, the spindown

\footnotetext{
${ }^{1}$ The most recent version is available at http://pulsar.princeton.edu/tempo, which is maintained and distributed by Princeton University and the Australia Telescope National Facility.
} 

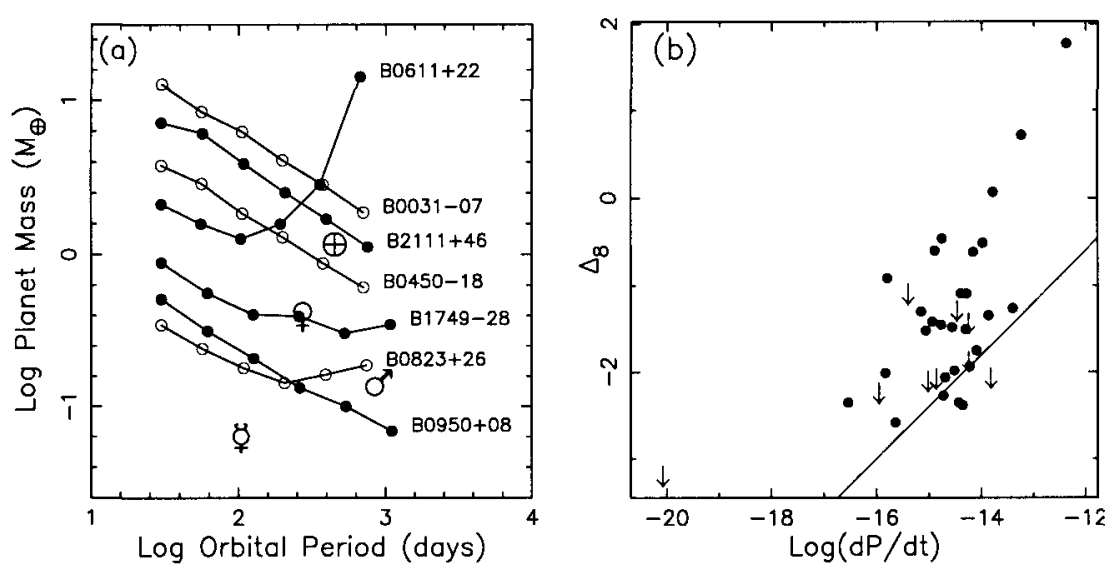

Figure 1. (a) Limits on planets around 7 pulsars from the TCfA sample. Locations of terrestrial planets are included for comparison purposes. (b) Timing activity of 38 pulsars timed at the TCfA. The solid line indicates an empirical relationship derived by Arzoumanian et al.

rate and, whenever necessary, the pulsar's astrometric parameters. The fits were performed for time spans of the data ranging from 30 days to two years. Clearly, the ability of the timing method to detect pulsar planets is limited by the pulse jitter and the instrumental noise for short orbital periods, and it stops improving as the time span of observations approaches a characteristic timescale of the timing noise for a given pulsar.

We have determined the basic timing noise characteristics of 38 pulsars by including the second-order period derivatives in the timing model fitting process and using their best-fit values to calculate a timing activity of each pulsar by means of the $\Delta_{8}$ parameter proposed by Arzoumanian et al. (1994). In Fig. 1 b, our results are compared to the empirical relationship, $\Delta_{8}=6.6+0.6 \log \dot{P}$, obtained by these authors. As in all the previous analyses of this kind (see also Dewey \& Cordes 1989), our data indicate a general decrease of the timing activity with the decreasing spindown rate of the pulsar.

A more detailed analysis of the TCfA timing observations of the complete set of program sources will be published in the forthcoming paper.

\section{References}

Arzoumanian, Z., Nice, D. J. \& Taylor, J. H., 1994, ApJ, 422, 671

Cordes, J. M., 1993, in Planets around Pulsars, ed. J. A. Phillips, S. E. Thorsett and S. R. Kulkarni, ASP Conf. Ser. 36, 43

Dewey, R. J. \& Cordes, J. M., 1989, NATO ASI: Timing Neutron Stars, 119

Konacki, M., Lewandowski, W., Wolszczan, A., Doroshenko, O. \& Kramer, M., ApJ, 519, L81 